\title{
Promoter methylation of tumor suppressor genes induced by human papillomavirus in cervical cancer
}

\author{
PATTAMAWADEE YANATATSANEEJIT ${ }^{1,2}$, KANWALAT CHALERTPET $^{3}$, JUTHAMARD SUKBHATTEE $^{1}$, \\ IRIN NUCHCHAROEN ${ }^{1}$, PIYATHIDA PHUMCHAROEN ${ }^{1}$ and APIWAT MUTIRANGURA ${ }^{2}$ \\ ${ }^{1}$ Human Genetics Research Group, Department of Botany, Faculty of Science, Chulalongkorn University; \\ ${ }^{2}$ Center of Excellence in Molecular Genetics of Cancer and Human Diseases, Department of Anatomy; \\ ${ }^{3}$ Inter-Department of Biomedical Sciences, Faculty of Medicine, Chulalongkorn University, Bangkok 10330, Thailand
}

Received August 1, 2019; Accepted January 14, 2020

DOI: $10.3892 / \mathrm{ol} .2020 .11625$

\begin{abstract}
Cervical cancer is the most fourth common cancer in women worldwide. The E6 and E7 high-risk human papillomavirus (HPV) types are the main cause of this cancer. Several studies have revealed that promoter methylation of tumor suppressor genes is induced by HPV E7. Recently, it was found that HPV16-E7 and the DNA methyltransferase 1 complex could bind at the cyclin A1 (CCNA1) promoter, resulting in $C C N A 1$ promoter methylation. Therefore, there is a need to study other tumor suppressor genes for which HPV may induce promoter methylation. The present study investigated whether HPV induced cell adhesion molecule 1 $(C A D M 1)$ and death associated protein kinase 1 (DAPK1) promoter methylation. C33a (no HPV infection) and $\mathrm{SiHa}$ (HPV 16 infection) cell lines were used for methylation status and expression observation. It was found that $C A D M 1$ and $D A P K 1$ promoter methylation, no expression of $C A D M 1$ and decreased expression of $D A P K 1$, was presented in $\mathrm{SiHa}$ cells. While no promoter methylation of these two genes was observed in C33a cells, with positive expression of the genes. It was subsequently investigated whether E6 and/or E7 could induce promoter methylation and decrease the expression of these two genes. Methylation-specific primer PCR and quantitative PCR were performed to elucidate the promoter methylation status and expression of $C A D M 1$ and $D A P K 1$ in C33a cells transfected with HPV16 E6-PCDNA3 or HPV16 E7-PCDNA3.1 myc-his, compared to empty vector-transfected cells. The results showed that HPV E7 could induce CADMI promoter methylation and decrease the gene expression in
\end{abstract}

Correspondence to: Dr Pattamawadee Yanatatsaneejit, Human Genetics Research Group, Department of Botany, Faculty of Science, Chulalongkorn University, 254 Payathai Road, Bangkok 10330, Thailand

E-mail: pattamawadee.y@chula.ac.th

Key words: promoter methylation, cell adhesion molecule 1, death associated protein kinase 1, human papillomavirus E6, human papillomavirus E7, cervical cancer
HPV E7 transfected C33a cells, while HPV E6 could induce $D A P K 1$ promoter methylation and decrease its expression in C33a cells transfected with HPV E6. Finally, the mechanism by which HPV E7 induced $C A D M 1$ promoter methylation was observed by performing chromatin immunoprecipitation; the data showed that E7 induced $C A D M 1$ methylation by the same mechanism as that for $C C N A 1$, by binding at the $C A D M 1$ promoter, resulting in the subsequent reduction of its expression in cervical cancer.

\section{Introduction}

Cervical cancer is one of the most common cancer types in women worldwide (1). In developing countries, cervical cancer is a major health problem. In Thailand, cervical cancer is one of five cancer types leading to the death of 7 Thai women per day (2). Several studies have identified risk factors for cervical cancer. Human papillomavirus (HPV) infection is the most important risk factor for cervical cancer (3). HPV can be divided into low-risk and high-risk groups. High-risk HPV types, including HPV16, 18 and 56, are those with high oncogenic potential that can be the cause of neoplastic transformation (4). The mechanism by which high-risk HPV leads to cervical cancer is still under investigation. The role of E6 and E7 in p53 and pRB degradation, respectively, is well known in cancer transformation (5). The role of E6 and E7 proteins in tumorigenesis is still being explored. Recent studies have illustrated that not only the degradation of p53 and pRB, but also the function of these two oncoproteins, are involved in the promoter methylation of tumor suppressor genes, resulting in tumorigenesis $(6,7)$. Our previous study found that cervical cancer tissues infected with the integrated form of either HPV 16 or 18 can exhibit methylation at the cyclin A1 (CCNAI) promoter (8). HPV is composed of 8 genes: $L 1, L 2, E 1, E 2, E 4$, $E 5, E 6$ and $E 7$. The integrated form of HPV exhibits overexpression of E6 and E7 because of E2 disruption. E6 and E7 are oncoproteins that cause cervical cancer and head and neck cancer (9).

There is much evidence showing the association between HPV infection and promoter methylation in cancer. A study by Sator et al (10) showed a higher level of promoter methylation and lower gene expression levels of seventy-five genes 
including IRS1, GNAI1, GNAI2, EREG, CCNA1, RGS4, and $P K I G$ resulting from HPV infection in head and neck cancer. A study by Lechner et al (11) showed increased mRNA expression of both DNA methyltransferase $3 \alpha($ Dnmt $3 a)$ and DNA methyltransferase 1 (Dnmt1) in HPV-positive head and neck cancer cell lines. Moreover, they observed that promoter methylation increased in E6 and E7-transfected head and neck cancer cell lines (10). A study by Kitkumthorn et al (12) demonstrated that CCNAl promoter methylation was detected in cervical intraepithelial neoplasia (CIN) grade III and invasive cancer related to HPV.

Promoter methylation of genes is a good biomarker for identifying women who are at risk of cervical cancer development. CCNAl promoter methylation is an efficient biomarker that can be diagnosed from precancerous stages to invasive cervical cancer (13). Our previous study found that HPV16-E7 can induce $C C N A 1$ promoter methylation by forming a complex with Dnmtl at the CCNA1 promoter (14). Therefore, there is a need to identify genes in which HPV can induce promoter methylation, in addition to CCNA1. Herein, not only HPV-E7 but also HPV-E6 were included for the assessment of their methylation ability. The promoter methylation and expression of two tumor suppressor genes, cell adhesion molecule 1 (CADMI) and death associated protein kinase 1 (DAPK1), were then observed in E6 and E7-transfected cervical cancer cell lines: C33a, which is HPV-negative; and $\mathrm{SiHa}$, which is HPV 16-positive. The studies of Steenbergen et al and Banzai et al found $C A D M 1$ and DAPK1 promoter methylation in HPV-related cervical cancer, respectively $(15,16)$. CADM1 is involved in cell adhesion, while $D A P K 1$ is involved in apoptosis (17). Promoter methylation of these genes suppresses their expression, leading to carcinogenesis. The aim of the present study was to investigate promoter methylation of $C A D M 1$ and $D A P K 1$ in the cells with HPV-E6 or E7 transfection together with the mechanism by which HPV-E7 induced promoter methylation of the genes.

\section{Materials and methods}

Cell culture. The SiHa (HPV 16-positive) cell line was kindly provided by Dr.Silvio Gutkind (Moores Cancer Center, UCSD, USA) with proof that there was no contamination, and the C33a (HPV-negative) cell line was purchased from the American Type Culture Collection (HTB-31TM; lot no. 63596879). The cells were grown and maintained in DMEM (Sigma-Aldrich; Merck KGaA) supplemented with 10\% FBS (Gibco; Thermo Fisher Scientific, Inc.) and 1\% antibiotic-antimycotic (Gibco; Thermo Fisher Scientific, Inc.) at $37^{\circ} \mathrm{C}$ in an atmosphere of $5 \% \mathrm{CO}_{2}$.

Recombinant plasmid. The E6 recombinant plasmid was inserted into a PCDNA3 vector, which was provided by Assoc. Prof. Dr. Andrew Yeudal (Augusta University, USA). The E7 recombinant plasmid was inserted into PCDNA3.1 myc-his, which was constructed as per a previous study (13). Both recombinant plasmids were sent for sequencing to confirm that the sequences were correct.

Transfection. E6 and $E 7$ recombinant plasmids were transfected into C33a cells. C33a cells were seeded into 6-well plates at $3 \times 10^{5}$ cells $/ \mathrm{ml}$ and incubated overnight. Next, $2 \mu \mathrm{g}$
E6 or E7 recombinant plasmid and pcDNA 3.1/myc-his (PC) empty plasmid (Invitrogen; Thermo Fisher Scientific, Inc.) were transfected using Lipofectamine ${ }^{\circledR} 2000$ (Thermo Fisher Scientific, Inc.), according to the manufacturer's protocol. After $72 \mathrm{~h}$, transfected cells were collected to study E6- and E7-mediated $C A D M 1$ and $D A P K 1$ promoter methylation, and $C A D M 1$ and DAPK1 mRNA expression. Moreover, cells transfected with E7 were harvested for chromatin immunoprecipitation. The transfection was performed in triplicate for all experiments.

Isolation of DNA. SiHa, C33a and C33a cells transfected with $E 6$ or $E 7$ were subjected to DNA extraction. Briefly, cells were digested with lysis buffer II containing SDS (Sigma-Aldrich; Merck $\mathrm{KGaA}$ ) and proteinase $\mathrm{K}$ (USB) at $50^{\circ} \mathrm{C}$ overnight. Phenol/chloroform extraction and ethanol precipitation were then carried out as previously described (14).

Preparation of RNA. SiHa , C33a and C33a cells transfected with $E 6$ or $E 7$ and empty vector were subjected to RNA extraction. Total RNA was extracted using the TRIzol ${ }^{\circledR}$ reagent (Invitrogen; Thermo Fisher Scientific, Inc.). Then, $5 \mu \mathrm{g}$ total RNA from each sample was synthesized to cDNA using the RevertAid first-strand cDNA synthesis kit, according to the manufacturer's specifications (Thermo Fisher Scientific, Inc.).

Sodium bisulfite treatment and methylation-specific PCR. A total of $750 \mathrm{ng}$ DNAsample was subjected to bisulfite treatment using the EZ DNA Methylation-Gold kit (Zymo Research Corp.), according to the protocol provided by the manufacturer. The eluted DNA was subsequently used to carry out methylation-specific PCR using $0.3 \mu \mathrm{M}$ methylated and unmethylated specific primers (Table I). The DNA was initially denatured for $15 \mathrm{~min}$ at $95^{\circ} \mathrm{C}$, followed by 35 cycles of $1 \mathrm{~min}$ at $95^{\circ} \mathrm{C}, 1 \mathrm{~min}$ at $60^{\circ} \mathrm{C}$ for $C A D M 1$ and $53^{\circ} \mathrm{C}$ for $D A P K 1$, and $1 \mathrm{~min}$ at $72^{\circ} \mathrm{C}$, and $72^{\circ} \mathrm{C}$ for $7 \mathrm{~min}$. Then, $10 \mu \mathrm{l}$ PCR products were observed by gel electrophoresis on $8 \%$ acrylamide gels and stained with SYBR (Lonza Group, Ltd.). The methylated and unmethylated band intensities of each sample were visualized and measured using Storm840 and ImageQuant Software (Amersham Biosciences; GE Healthcare). The experiment was performed in triplicate.

Expression analysis. Quantitative PCR was performed to observe the DAPK1 and CADM1 mRNA expression in $\mathrm{SiHa}$ C33a cells and c33a transfected cells. Amplification was performed with $0.1 \mu \mathrm{M} \mathrm{DAPK1}$ and $0.1 \mu \mathrm{M} \mathrm{CADM1}$ primers (Table I), including primers for $0.1 \mu \mathrm{M} G A P D H$, which was used as a reference gene, and 19 Power SYBR Green PCR master mix (Applied Biosystems; Thermo Fisher Scientific, Inc.). The cDNA was amplified using a 7500-fast real-time PCR system (Applied Biosystems; Thermo Fisher Scientific, Inc.) with initially denatured for $10 \mathrm{~min}$ at $95^{\circ} \mathrm{C}$, followed by 35 cycles of $1 \mathrm{~min}$ at $95^{\circ} \mathrm{C}, 1 \mathrm{~min}$ at $60^{\circ} \mathrm{C}$, and $1 \mathrm{~min}$ at $72^{\circ} \mathrm{C}$, and $72^{\circ} \mathrm{C}$ for $7 \mathrm{~min}$. The fold changes in expression of $D A P K 1$ and $C A D M 1$ between experimental and control cellswere then determined using the ${ }^{\Delta \Delta} \mathrm{Ct}$ method (18). The experiment was performed in triplicate.

Chromatin immunoprecipitation. Chromatin immunoprecipitation was carried out in E7 recombinant 
Table I. Primer sequences, amplicon sizes and annealing temperature and conditions for PCR analysis.

\begin{tabular}{|c|c|c|c|}
\hline Primers & Sequence $\left(5^{\prime}-3^{\prime}\right)$ & Amplicon sizes, bp & Annealing temperature, ${ }^{\circ} \mathrm{C}$ \\
\hline \multicolumn{4}{|l|}{ MSP } \\
\hline \multicolumn{4}{|l|}{ CADM1 } \\
\hline CADM1-met (F) & GCGTCGTCGAACGTTAGCG & 165 & 60 \\
\hline CADM1-met (R) & GTTAACTACCTCCGAAACCCG & & \\
\hline CADM1-unmet (F) & TGAATGTTAGTGTTAGGGGGTG & 72 & 60 \\
\hline CADM1-unmet (R) & CACCACAAACCCAACCCAAC & & \\
\hline \multicolumn{4}{|l|}{$D A P K 1$} \\
\hline DAPK1-met (F) & CGAGCGTCGCGTAGAATT & 114 & 53 \\
\hline DAPK1-met (R) & CGAAAAACGACCGACAAACG & & \\
\hline DAPK1-unmet (F) & TGAGTTTGGAGTGTGGAGTT & 96 & 53 \\
\hline DAPK1-unmet (R) & AACACAACCCACCCACCT & & \\
\hline \multicolumn{4}{|l|}{$C A D M 1$ expression } \\
\hline CADM1 (F) & TGACAGTGATCGAGGGAGAGGT & 236 & 60 \\
\hline CADM1 (R) & GGGATCGGTATAGAGCTGGCAA & & \\
\hline \multicolumn{4}{|l|}{$D A P K 1$ expression } \\
\hline DAPK1 (F) & CCACCACTCGGATCAAGATCATTG & 132 & 60 \\
\hline DAPK1 (R) & ATATCTGCCTCAAGACCAAGAGG & & \\
\hline \multicolumn{4}{|l|}{$G A P D H$ expression } \\
\hline GAPDH (F) & GTGGGCAAGGTATCCCTG & 90 & 60 \\
\hline GAPDH (R) & GATTCAGTGTGGTGGGGGAC & & \\
\hline \multicolumn{4}{|l|}{ CADM1 ChIP-PCR } \\
\hline CADM1ChIP (F) & ACTCCGCCTCCAGCGCATGT & 229 & 62 \\
\hline CADM1ChIP (R) & CCCACACCTACCTGTGGGGAT & & \\
\hline
\end{tabular}

F, forward; R, reverse; met, methylated; unmet, unmethylated; ChIP, chromatin immunoprecipitation; CADM1, cell adhesion molecule 1; $D A P K 1$, death associated protein kinase 1.

plasmid- and empty plasmid-transfected C33a cells, as previously described, with some modifications (19). To observe the binding of E7 protein (with or without the CR3 region) at the $C A D M 1$ promoter, the chromatin fragments were immunoprecipitated with $10 \mu \mathrm{g}$ of the H3K4 (Abcam (Cambridge, UK), HPV16-E7 (Santa Cruz, CA, USA) and IgG (Santa Cruz, CA, USA) antibodies. Next, the precipitated DNA was further analyzed by PCR using $0.3 \mu \mathrm{M}$ $C A D M 1-C h I P F$ and $0.3 \mu \mathrm{M} C A D M 1-C h I P R$ (Table I) with initially denatured for $10 \mathrm{~min}$ at $95^{\circ} \mathrm{C}$, followed by 35 cycles of $1 \mathrm{~min}$ at $95^{\circ} \mathrm{C}, 1 \mathrm{~min}$ at $62^{\circ} \mathrm{C}$, and $1 \mathrm{~min}$ at $72^{\circ} \mathrm{C}$, and $72^{\circ} \mathrm{C}$ for $7 \mathrm{~min}$. Then, $10 \mu 1 \mathrm{PCR}$ products were observed by gel electrophoresis using a $8 \%$ acrylamide and $1 \%$ agarose gel stained with SYBR (Lonza Group, Ltd.). The CADM1 bands were visualized using Storm840 and ImageQuanNT Software (Amersham Biosciences; GE Healthcare). The experiment was performed in triplicate.

Sequencing analysis. To confirm the accuracy of the E6 and $E 7$ recombinant plasmid sequences, E6 and E7 recombinant plasmids were transformed into competent Escherichia coli XL-1 blue cells for cloning, and then the plasmids were extracted using a GeneJET Plasmid Miniprep kit (Fermentas;
Thermo Fisher Scientific, Inc.) for sequencing analysis immediately after transfection. After alignment between the sequence of recombinant plasmid and GENBANK (www. ncbi.nlm.nig.gov), the accuracy of the $E 6$ and $E 7$ sequences were confirmed.

Statistical analysis. To test the significance of the E6- or E7-mediated induction of $C A D M 1$ and $D A P K 1$ promoter methylation and the decrease in gene expression, two sample t-tests between two groups of samples, E6 or E7 transfected cells and control cells, were used. $\mathrm{P}<0.05$ was considered to indicate a statistically significant difference.

\section{Results}

Methylation and expression status in $H P V+$ and $H P V$-cervical cancer cell lines. To investigate the effect of HPV in mediating promoter methylation and decreasing gene expression, the SiHa cervical cancer cell line with HPV type 16 infection and the $\mathrm{C} 33$ a cervical cancer cell line without HPV infection were used for the observation of methylation and the expression status of $C A D M 1$ and $D A P K 1$. There was methylation at the $C A D M 1$ and $D A P K 1$ promoters in SiHa cells, whereas there 
A

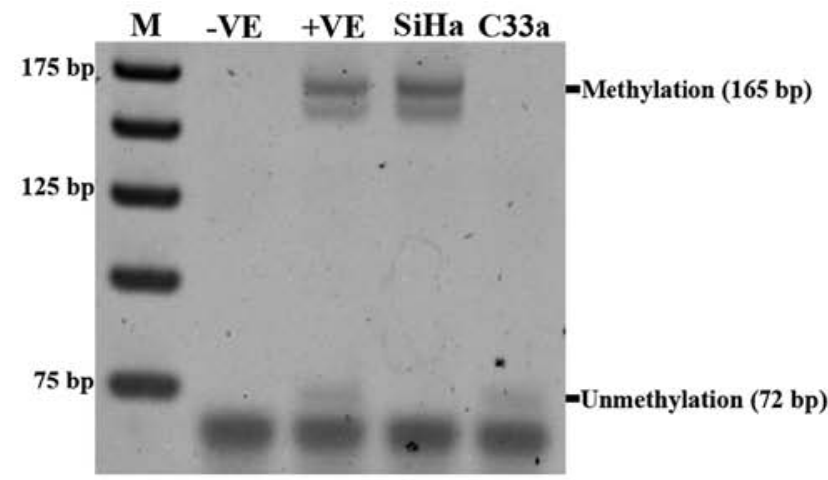

C

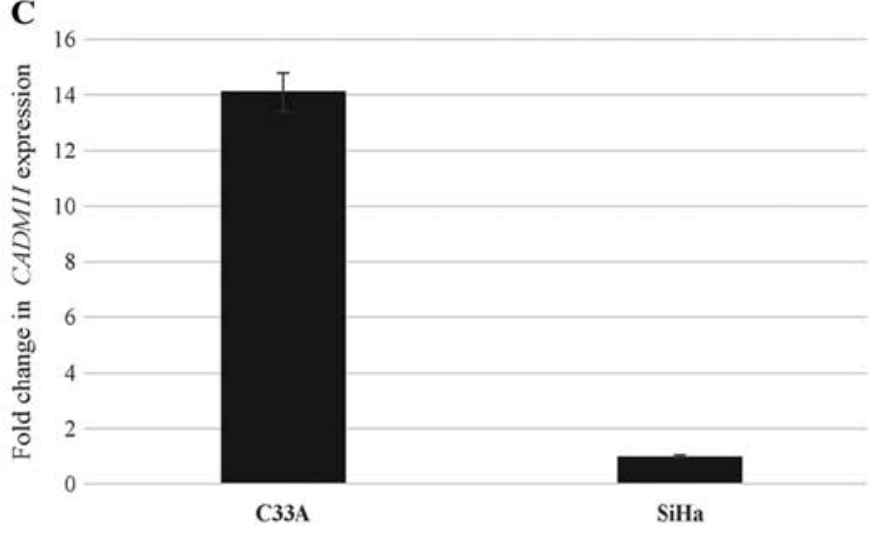

B

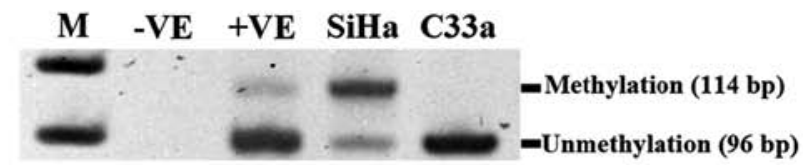

D

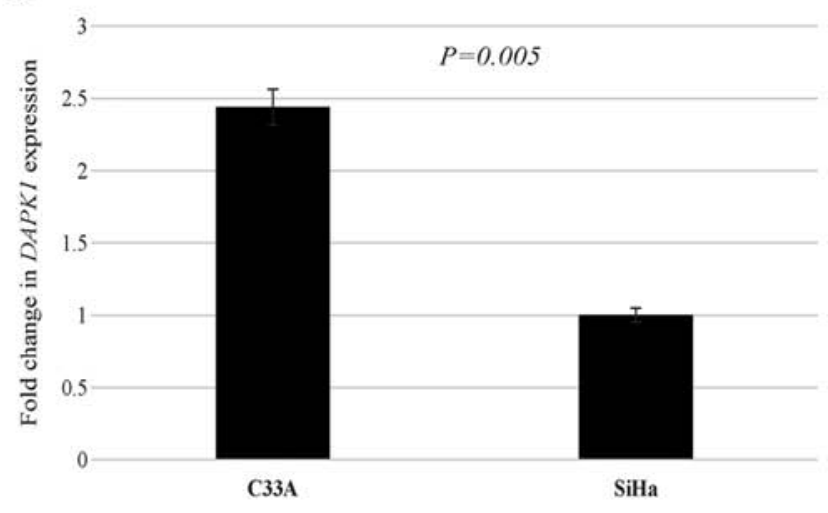

Figure 1. Profile of promoter methylation and the expression of $C A D M 1$ and $D A P K 1$ in SiHa and C33a cell lines: Methylation status of (A) CADM1 promoter and (B) DAPK1 promoter was detected by methylation-specific PCR using duplex primers for methylated and unmethylated regions. Expression status of (C) CADM1 and (D) DAPK1 in C33a and SiHa was investigated by quantitative PCR. -ve, distilled water; +ve, mixture of human methylated and non-methylated DNA. $C A D M 1$, cell adhesion molecule 1; $D A P K 1$, death associated protein kinase 1.

was no methylation at the $C A D M 1$ and $D A P K 1$ promoters in $\mathrm{C} 33 \mathrm{a}$ cells (Fig. 1A and B). There was no expression of $C A D M 1$ in SiHa cells, while the expression of this gene in C33a cells was detected. For $D A P K 1$, significantly decreased expression was detected in $\mathrm{SiHa}$ cells compared to that in C33a cells; $\mathrm{P}=0.005$. (Fig. $1 \mathrm{C}$ and $\mathrm{D}$ ).

HPV E6 and E7 induces DAPK1 and CADM1 promoter methylation and decreases their expression. To investigate whether E6 or E7 of HPV induce de novo methylation of $D A P K 1$ and $C A D M 1, E 6$ or $E 7$ recombinant plasmids and empty PCDNA3.1 (as a control) were successfully transfected into $\mathrm{C} 33 \mathrm{a}$ cell (Fig. $2 \mathrm{~A}$ and B). CADM1 and $D A P K 1$ promoter methylation and their expression were observed. For methylation status in E6- or E7-transfected cells compared to control cells, significant $C A D M 1$ promoter methylation was observed in E7-transfected cells compared with control cells $(\mathrm{P}<0.0001)$, while the promoter methylation of this gene could not be detected in C33a cells transfected with E6. To observe the methylation of $D A P K 1$ in C33a-transfected cells, the results showed that significantly increased $D A P K 1$ promoter methylation was detected in E6-transfected cells compared to cells with the empty vector $(\mathrm{P}=0.0003)$, whereas there was no $D A P K 1$ methylation in E7-transfected cells (Fig. 3A and B). For the expression study, after $E 7$-transfected into C33a cells, there was a significantly decreased $C A D M 1$ expression in E7-transfected C33a cells $(\mathrm{P}=0.008)$ compared to cells with the empty vector. To investigate the expression of $D A P K 1$, after E6 and empty vector transfection into $\mathrm{C} 33 \mathrm{a}$ cells, there was significantly decreased expression of DAPK1 $(\mathrm{P}=0.001)$ in E6-transfected cells compared to cells with the empty vector (Fig. 3C and D).

E7 targets the CADM1 promoter. It was hypothesized that E7 promotes $C A D M 1$ methylation by forming a complex with Dnmt1 at the $C A D M 1$ promoter, which is the same mechanism as that for CCNA1. To demonstrate that this hypothesis was viable, a chromatin immunoprecipitation assay was carried out in C33a cells transfected with E7 and the empty vector by precipitating with anti-HPV16 E7 and performing PCR to obtain a 229-bp CADM1 product. As shown in Fig. 4, the product band of the $C A D M 1$ promoter was detected in E7-overexpressing C33a cells, but not in the cells with the empty vector. These data suggested that $\mathrm{E} 7$ can bind at the $C A D M 1$ promoter.

\section{Discussion}

Epigenetics plays an important role in tumorigenesis. DNA methylation at the promoter of tumor suppressor genes is 
A 200

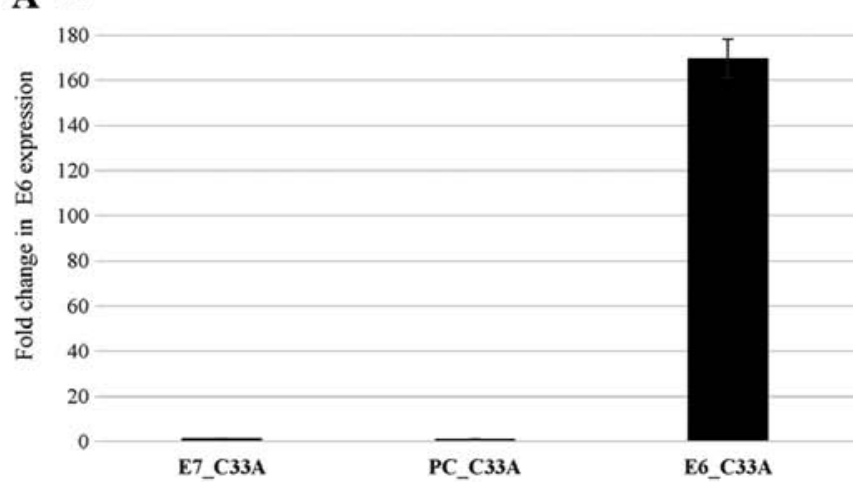

B

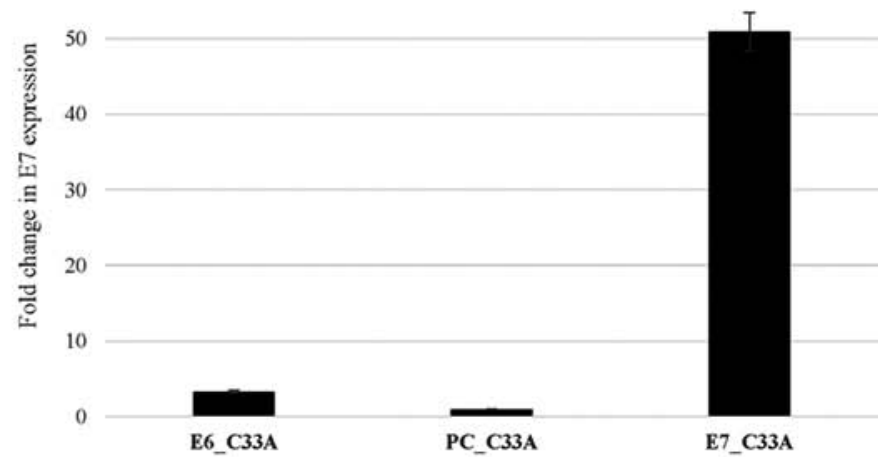

Figure 2. Expression of $E 6$ and $E 7$ in E6 or E7 recombinant plasmid and empty vector (PC) transfected cells. (A) E6 expression was observed in three transfected cells; E7_C33A was E7 transfected C33A cells, PC_C33A was PC transfected C33A cells, E6_C33A was E6 transfected C33A cells. (B) E7 expression was observed in three transfected cells; E6_C33A was E6 transfected C33A cells, PC_C33A was PC transfected C33A cells, E7_C33A was E7 transfected C33A cells.

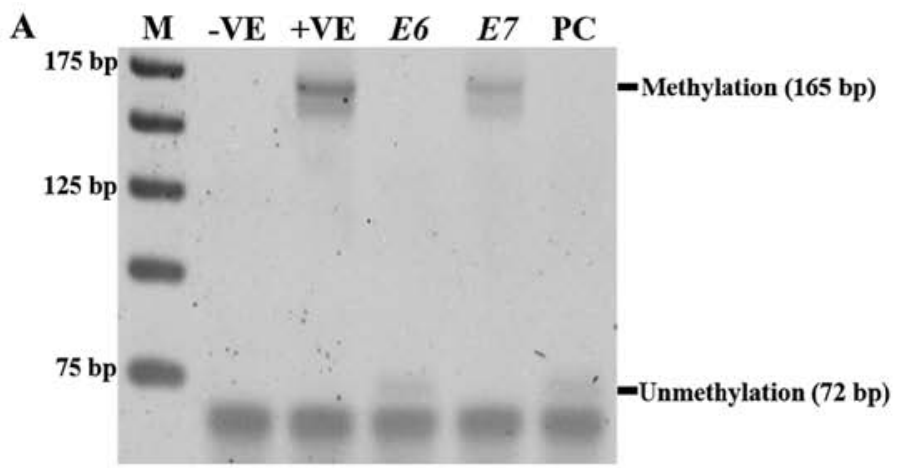

$\mathbf{B}$

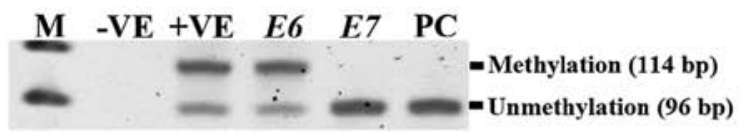

C

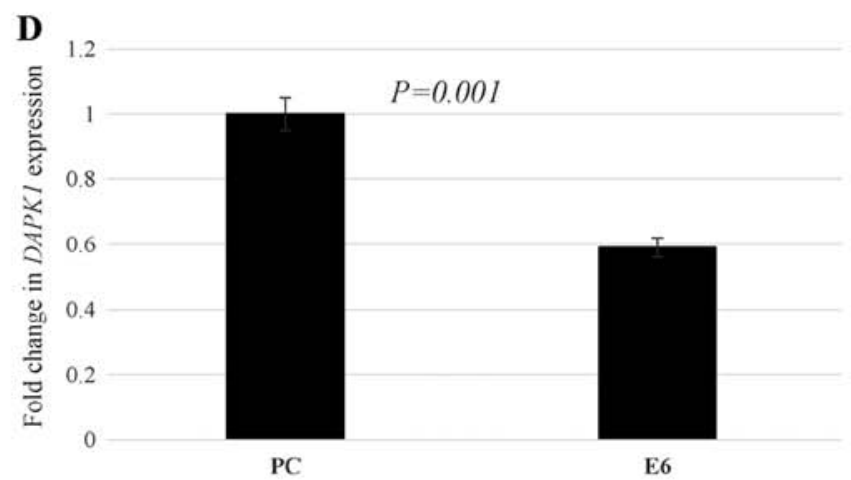

Figure 3. Promoter methylation and the expression of $C A D M 1$ and $D A P K 1$ were investigated in E6 or E7 recombinant plasmid and empty vector (PC)-transfected cells. Methylation status of (A) CADM1 and (B) DAPK1 was observed; -ve was distilled water, +ve was the mixture of human methyled and non-methylated DNA, E6, E7 and PC were E6, E7 and PC (empty vector) transfected cells, respectively. The expression of (C) CADM1 in C33a cells transfected with E7 and PC (empty vector) (D) DAPK1 in C33a cells transfected with E6 and PC (empty vector). CADM1, cell adhesion molecule 1; DAPK1, death associated protein kinase 1.

a process that decrease their expression, resulting in the development of many cancer types, including cervical cancer. In addition to promoter methylation, HPV is also a major cause of cervical cancer. To date, there have been several studies showing that HPV induces promoter methylation of tumor suppressor genes. It has been found that E6 and E7 can promote the promoter methylation of several genes. For example, a study by Li et al (20) reported that E6 and E7 gene silencing led to a decrease in the methylation of six tumor suppressor genes, MTIG, NMES1, RRAD, SFRP1, SPARC and TFP12, and induced the phenotypic transformation of human cervical carcinoma cell lines.

The mechanism by which E6 or E7 could induce the promoter methylation of the genes has been explored. Our 


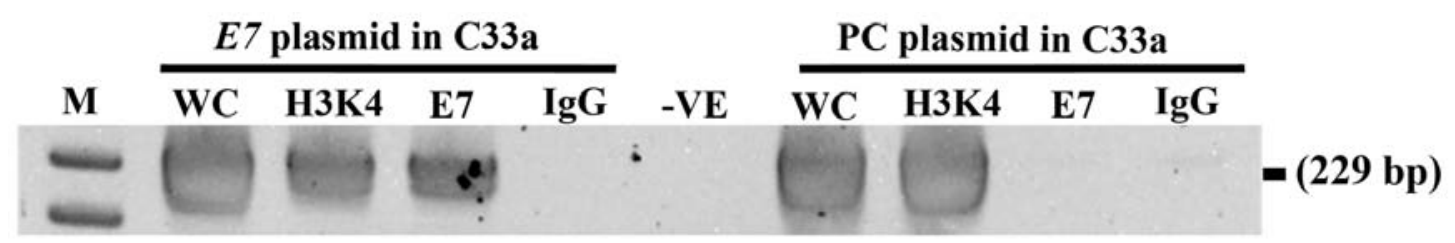

Figure 4. E7 and PC (empty vector) were transfected in C33a cells. Chromatin immunoprecipitation was used to confirm the binding of the E7 at the $C A D M 1$ promoter. WC was whole cell lysate used as a positive control for PCR. H3K4 was used as a positive control antibody for the ChIP assay. E7 antibody was used to observe the binding of E7 at $C A D M 1$ promoter. IgG served as negative control antibody for ChIP. CADM1, cell adhesion molecule 1; DAPK1, death associated protein kinase 1; ChIP, chromatin immunoprecipitation.

previous study demonstrated that HPV 16 E7 had an interaction with Dnmt1 resulting in $C C N A 1$ promoter methylation and a reduction in expression (14). A study by Au Yeung et al (21) indicated that E6 increased the expression of Dnmt1 by degrading p53. Dnmt1 is a member of the Dnmt family, which can play role in de novo methylation (22). Therefore, the present study sought to discover genes whose promoter methylation may be induced by E6 or E7. Here, $C A D M 1$ and $D A P K 1$ were selected to be candidate genes because of their functions as tumor suppressor genes, and the evidence showing that the methylation of these two genes may be related to cervical cancer progression. CADMI is hypermethylated in CIN II-III and cervical cancer, while $D A P K 1$ is hypermethylated in CIN III and cervical cancer (23). Notably, it was found that the promoter methylation of both genes was induced by HPV. Nevertheless, HPV16 E6 only induced methylation of DAPK1, while HPV 16 E7 only induced methylation of $C A D M 1$.

The present study demonstrated that HPV 16 E7 can induce promoter methylation of $C A D M 1$, and not only of $C C N A 1$. Therefore, it was hypothesized that the mechanism by which HPV16 E7 induced promoter methylation of CADM1 may be the same as the mechanism for CCNA1. To elucidate this mechanism, chromatin immunoprecipitation was carried out using an E7 antibody to precipitate E7 protein binding at the $C A D M 1$ promoter. The results showed that $\mathrm{E} 7$ protein can bind at the $C A D M 1$ promoter. Studies by Chalertpetch et al (14) and Burgers et al (24) found an interaction between E7 and Dnmt1. This evidence indicated that that HPV16 E7 may bind with Dnmt1 at the $C A D M 1$, promoter leading to aberrant promoter methylation and decreasing the expression of the gene.

In the cells, it was hypothesized that E6 and E7 may work together to induce promoter methylation. E6 was thought to increase Dnmt1 expression (21), and E7 to bind with Dnmt1 and target the promoters (14). However, it was found that DAPK1 promoter methylation can be augmented by E6, but not by E7. Therefore, it is possible that E6 might increase Dnmtl activity and thus has another mechanism through which it targets $D A P K 1$ to induce its promoter methylation. The mechanism underlying the increase in $D A P K 1$ promoter methylation by E6 remains to be studied. However, in E7-transfected cells, $C A D M 1$ promoter methylation was observed. This may be because there is endogenous Dnmt1 in the cells, thus E7 protein can form complexes with endogenous Dnmt1 and induce $C A D M 1$ promoter methylation.

Understanding the mechanism by which HPV induces promoter methylation of genes will be useful for drug discovery and clinical diagnosis. At present, a Pap smear is still the gold standard for cervical cancer diagnosis.
Nevertheless, there is a chance of receiving false negative results from a Pap smear test $(25,26)$. In addition, Pap smear tests frequently detect atypical squamous cells of undetermined significance (ASCUS) (27). Therefore, molecular marker findings, together with HPV testing, may be a good alternative for diagnosing the early stages of cervical cancer, eventually leading to a decrease in the number of cervical cancer patients worldwide.

\section{Acknowledgements}

Not applicable.

\section{Funding}

The present study was financially supported by Thailand Research Fund and Chulalongkorn University (grant no. RSA5880065) and The National Science and Technology Development Agency, Thailand (grant no P-15-50270).

\section{Availability of data and materials}

The datasets used and/or analyzed during the present study are available from the corresponding author on reasonable request.

\section{Authors' contributions}

PY wrote the proposal for grants, designed the experiments, analyzed data and wrote the manuscript. $\mathrm{KC}$ performed the experiments and analyzed data. JS performed the experiments and analyzed data. IN performed the experiments and analyzed data. PP performed the experiments and analyzed data. AM designed the experiments. All authors read and approved the final manuscript.

\section{Ethics approval and consent to participate}

Not applicable.

\section{Patient consent for publication}

Not applicable.

\section{Competing interests}

The authors declare that they have no competing interests. 


\section{References}

1. Arbyn M, Castellsagué X, de Sanjosé S, Bruni L, Saraiya M, Bray F and Ferlay J: Worldwide burden of cervical cancer in 2008. Ann Oncol 22: 2675-2686, 2011.

2. Virani S, Bilheem S, Chansaard W, Chitapanarux I, Daoprasert K, Khuanchana S, Leklob A, Pongnikorn D, Rozek LS, Siriarechakul S, et al: National and subnational population-based incidence of cancer in thailand: Assessing cancers with the Highest Burdens. Cancers (Basel) 9: pii: E108, 2017.

3. Bosch FX, Burchell AN, Schiffman M, Giuliano AR, de Sanjose S, Bruni L, Tortolero-Luna G, Kjaer SK and Muñoz N: Epidemiology and natural history of human papillomavirus infections and type-specific implications in cervical neoplasia. Vaccine 26 (Suppl 10): K1-K16, 2008.

4. Tulay P and Serakinci N: The role of human papillomaviruses in cancer progression. J Cancer Metastasis Treat 2: 201-213, 2016.

5. Yeo-Teh NSL, Ito Y and Jha S: High-risk human papillomaviral oncogenes E6 and E7 target key cellular pathways to achieve oncogenesis. Int J Mol Sci 19: pii: E1706, 2018.

6. Sen P, Ganguly P and Ganguly N: Modulation of DNA methylation by human papillomavirus E6 and E7 oncoproteins in cervical cancer. Oncol Lett 15: 11-22, 2018.

7. Yin F, Wang N, Wang S, Yu F, Sun X, Yu X, Luo B, Zhao C and Wang Y: HPV16 oncogenes E6 or/and E7 may influence the methylation status of RASSFIA gene promoter region in cervical cancer cell line HT-3. Oncol Rep 37: 2324-2334, 2017.

8. Yanatatsaneejit P, Mutirangura A and Kitkumthorn N: Human papillomavirus's physical state and cyclin A1 promoter methylation in cervical cancer. Int J Gyneco 21: 902-906, 2011.

9. Chung CH and Gillson ML: Human papillomavirus in head and neck cancer: Its role in pathogenesis and clinical implications. Clin Cancer Res 15: 6758-6762, 2009.

10. Sartor MA, Dolinoy DC, Jones TR, Colacino JA, Prince ME, Carey TE and Rozek LS: Genome-wide methylation and expression differences in HPV(+) and HPV(-) squamous cell carcinoma cell lines are consistent with divergent mechanisms of carcinogenesis. Epigenetics 6: 777-787, 2011.

11. Lechner M, Fenton T, West J, Wilson G, Feber A, Henderson S, Thirlwell C, Dibra HK, Jay A, Butcher L, et al: Identification and functional validation of HPV-mediated hypermethylation in head and neck squamous cell carcinoma. Genome Med 5: 15, 2013.

12. Kitkumthorn N, Yanatatsanajit P, Kiatpongsan S, Phokaew C, Triratanachat S, Trivijitsilp P, Termrungruanglert W, Tresukosol D, Niruthisard S and Mutirangura A: Cyclin A1 promoter hypermethylation in human papillomavirus-associated cervical cancer BMC Cancer 6: 55, 2006.

13. Chujan S, Kitkumthorn N, Siriangkul S and Mutirangura A: CCNA1 promoter methylation: A potential marker for grading Papanicolaou smear cervical squamous intraepithelial lesions. Asian Pac J Cancer Prev 15: 7971-7975, 2014.

14. Chalertpet K, Pakdeechaidan W, Patel V, Mutirangura A and Yanatatsaneejit P: Human papillomavirus type 16 E7 oncoprotein mediates CCNA1 promoter methylation. Cancer Sci 106: 1333-1340, 2015.
15. Steenbergen RD, Kramer D, Braakhuis BJ, Stern PL, Verheijen RH, Meijer CJ and Snijders PJ: TSLC1 gene silencing in cervical cancer cell lines and cervical neoplasia. J Natl Cancer Inst 96: 294-305, 2004.

16. Banzai C, Nishino K, Quan J, Yoshihara K, Sekine M, Yahata T and Tanaka K; Gynecological Cancer Registry of Niigata: Promoter methylation of DAPK1, FHIT, MGMT, and CDKN2A genes in cervical carcinoma. Int J Clin Oncol 19: 127-132, 2014.

17. Yang HJ: Aberrant DNA methylation in cervical carcinogenesis. Chin J Cancer 32: 42-48, 2013.

18. Livak KJ and Schmittgen TD: Analysis of relative gene expression data using real-time quantitative PCR and the 2(-Delta Delta C(T)) method. Methods 25: 402-408, 2001.

19. Boyd KE and Farnham PJ: Coexamination of site-specific transcription factor binding and promoter activity in living cells. Mol Cell Biol 19: 8393-8399, 1999.

20. Li L, Xu C, Long J, Shen D, Zhou W, Zhou Q, Yang J and Jiang M: E6 and E7 gene silencing results in decreased methylation of tumor suppressor genes and induces phenotype transformation of human cervical carcinoma cell lines. Oncotarget 6: 23930-23943, 2015.

21. Au Yeung CL, Tsang WP, Tsang TY, Co NN, Yau PL and Kwok TT: HPV-16 E6 upregulation of DNMT1 through repression of tumor suppressor p53. Oncol Rep 24: 1599-1604, 2010.

22. Fatemi M1, Hermann A, Gowher H and Jeltsch A: Dnmt3a and Dnmtl functionally cooperate during de novo methylation of DNA. Eur J Biochem 269: 4981-4984, 2002.

23. Feng C, Dong J, Chang W, Cui M and Xu T: The progress of methylation regulation in gene expression of cervical cancer. Int J Genomics 2018: 8260652, 2018.

24. Burgers WA, Blanchon L, Pradhan S, de Launoit Y, Kouzarides T and Fuks F: Viral oncoproteins target the DNA methyltransferases. Oncogene 26: 1650-1655, 2007.

25. van der Graaf Y and Vooijs GP: False negative rate in cervical cytology. J Clin Pathol 40: 438-442, 1987.

26. Sprenger E, Schwarzmann P, Kirkpatrick M, Fox W, Heinzerling RH, Geyer JW and Knesel EA: The false negative rate in cervical cytology. Comparison of monolayers to conventional smears. Acta Cytol 40: 81-89, 1996.

27. Jahic $M$ and Jahic E: Diagnostic approach to patients with atypical squamous cells of undetermined significance cytologic findings on cervix. Med Arch 70: 296-298, 2016. 